\title{
Do Malaysian asthma patients use asthma action plan? a cross sectional study in Malaysian primary care setting
}

\begin{abstract}
Background: Supported self-management, including provision of an asthma action plan (AAP) improves asthma control. However, the concept of self-management and use of AAP among Asian population is relatively new. Aims: To determine the prevalence of AAP ownership among asthma patients and their characteristics in Malaysia primary care. Methods: We randomly sampled 550 asthma patients from five primary care practices between Oct 2017-Jan 2018. The patients were interviewed with pre-tested questionnaires to assess their AAP ownership, socio-demographic and the Asthma Control Test (ACT). Descriptive statistics were used to analyse the data. Results: Asthma control was poor in $143(26 \%)$ patients, partially controlled in $137(24.9 \%)$ and well-controlled in $270(29.1 \%)$. Only 160(29.1\%) owned an AAP. These AAP were primarily given by the doctors $(95 \%$, $\mathrm{n}=152$ ) and $5 \%$ were given by the allied health (nurses and pharmacists). Among those who owned an AAP, 106(66.2\%) were confident to use it. For those using AAP, the mean age was 45.6 years old, predominantly female (67\%), of Malay ethnicity (48\%), had at least secondary educational level (40\%) and had well-controlled asthma (46\%). Conclusion: Education, usage and confidence in using an AAP for asthma self-management is suboptimal among asthma patients in Malaysian primary care. Supported asthma selfmanagement in this population must be strengthened.
\end{abstract}

Keyword: Malaysian asthma patient; Asthma action plan; Asthma patient 